\title{
Transcriptome instability as a molecular pan-cancer characteristic of carcinomas
}

\author{
Anita Sveen ${ }^{1,2}$, Bjarne Johannessen ${ }^{1,2}$, Manuel R Teixeira ${ }^{2,3,4,5}$, Ragnhild A Lothe ${ }^{1,2}$ and Rolf I Skotheim ${ }^{1,2^{*}}$
}

\begin{abstract}
Background: We have previously proposed transcriptome instability as a genome-wide, pre-mRNA splicing-related characteristic of colorectal cancer. Here, we explore the hypothesis of transcriptome instability being a general characteristic of cancer.

Results: Exon-level microarray expression data from ten cancer datasets were analyzed, including breast cancer, cervical cancer, colorectal cancer, gastric cancer, lung cancer, neuroblastoma, and prostate cancer (555 samples), as well as paired normal tissue samples from the colon, lung, prostate, and stomach (93 samples). Based on alternative splicing scores across the genomes, we calculated sample-wise relative amounts of aberrant exon skipping and inclusion. Strong and non-random $(P<0.001)$ correlations between these estimates and the expression levels of splicing factor genes $(n=280)$ were found in most cancer types analyzed (breast-, cervical-, colorectal-, lung- and prostate cancer). This suggests a biological explanation for the splicing variation. Surprisingly, these associations prevailed in pan-cancer analyses. This is in contrast to the tissue and cancer specific patterns observed in comparisons across healthy tissue samples from the colon, lung, prostate, and stomach, and between paired cancer-normal samples from the same four tissue types.

Conclusion: Based on exon-level expression profiling and computational analyses of alternative splicing, we propose transcriptome instability as a molecular pan-cancer characteristic. The affected cancers show strong and non-random associations between low expression levels of splicing factor genes, and high amounts of aberrant exon skipping and inclusion, and vice versa, on a genome-wide scale.
\end{abstract}

Keywords: Alternative splicing, Carcinomas, Exon microarray, Splicing factor, Tissue specificity

\section{Background}

The four major types of cancer, lung cancer, breast cancer, colorectal cancer, and prostate cancer, constitute approximately $40 \%$ of cancer cases world-wide, with more than 5 million new diagnoses and 2.7 million related deaths every year [1]. These are all epithelial cancers and are commonly characterized by genomic instability [2]. Genomic instability is described as an enabling hallmark of cancer, generating random mutations which may also hit cancer critical genes [3]. At the nucleotide level, genomic instability can occur as frequent mutations of short nucleotide repeats dispersed throughout the

\footnotetext{
* Correspondence: rolfi.skotheim@rr-research.no

'Department of Cancer Prevention, Institute for Cancer Research, The

Norwegian Radium Hospital, Oslo University Hospital, P.O. Box 4953 Nydalen, Oslo NO-0424, Norway

${ }^{2}$ Centre for Cancer Biomedicine, Faculty of Medicine, University of Oslo, P.O. Box 1078 Blindern, Oslo NO-0316, Norway

Full list of author information is available at the end of the article
}

genome, referred to as microsatellite instability, and results from a defective mismatch repair system [4-6]. Microsatellite instability has been described in several solid cancer types, including cancers of the colon, endometrium, stomach, and lung [7-11], and is associated with good prognosis in colorectal cancer $[9,12,13]$. Chromosomal instability, characterized by numerical and structural chromosome changes, is common in solid cancers, but the causes for this molecular phenotype remain mostly unknown. Measured as aneuploidy, chromosomal instability has been found to be associated with poor prognosis in all four major types of carcinomas [14-17]. In addition to genomic instability, epigenome abnormality has also been described in several cancer types, although most prominent in colorectal cancer [18]. Cancers with the $\mathrm{CpG}$ island methylator phenotype have frequent DNA hypermethylation in gene promoter regions, often resulting in gene silencing [19]. Recently, we described 
genome-wide instability acting also on the level of the transcriptome in colorectal cancer, affecting the premRNA splicing process [20]. This transcriptome instability (TIN) was characterized by large variation in amounts of aberrant inclusion and skipping of exons in colorectal cancer samples. These amounts were shown to be strongly associated with both the expression levels of pre-mRNA splicing factors and poor prognosis for patients with colorectal cancer [20].

RNA splicing is a tightly regulated and highly tissue specific process that can occur by a number of different modes [21]. Alternative splicing modes include differential inclusion of whole exons (cassette exons) or parts of exons (alternative 5' or 3' splice sites), intron retention, mutual exclusion of cassette exons, alternative ordering of exons (exon scrambling) [22], and splicing of exons encoded by different genes (trans-splicing) [23]. Such alternative splicing is a major source of functional diversity in the human genome [24]. Nearly all multi-exon genes are alternatively spliced, predicted to undergo on average seven alternative splicing events during tissue development and across tissue types [25,26]. In cancer, the RNA splicing pathway is commonly disrupted, as evident by aberrant, disease-specific splicing patterns [27]. There is a large collection of proteins regulating the splicing process, and genes encoding these splicing factors have been identified as cancer critical genes, as is the case with SRSF1 [28]. Splicing factors have been found to be both differentially expressed [29] and commonly mutated in cancer [30-32]. Hence, there is tremendous potential for splicing variation in the cancer transcriptome.

In the present study, we describe TIN in several major types of malignancies, including breast cancer, cervical cancer, colorectal cancer, lung cancer, and prostate cancer. By analyzing exon microarray profiles for alternative splicing, we characterized the individual samples within the datasets for relative amounts of aberrant exon skipping and inclusion. In cancer types with TIN, we found strong and non-random associations between these amounts and the expression levels of splicing factors. Surprisingly, this association was intact in pan-cancer analysis of the TINcancers, indicating that the pronounced tissue specificity found in corresponding analyses across normal tissue types and paired cancer-normal samples was lost.

\section{Results}

Strong correlations between TIN-estimates and expression levels of splicing factors

Exon microarray profiles for 555 samples from ten different cancer datasets and 93 samples from four normal tissue datasets (Table 1) were analyzed for genome-wide alternative splicing variation. The lower and upper $1^{\text {st }}$ percentiles of alternative splicing (FIRMA) scores within each dataset were used as threshold values to designate exons as aberrantly spliced (range -2.4 to -2.0 and 1.8 to 2.1 , respectively; log2-scale; Table $\mathrm{S} 1$ in Additional file 1). Sample-wise amounts of aberrant exon skipping and inclusion were calculated as the number of exons exceeding these threshold values (Additional file 1: Table S1). These sample-wise amounts of aberrant splicing, relative to the average amount within the dataset, are referred to as TINestimates. The TIN-estimates and range of TIN-estimates (the difference between the sample with the highest and lowest estimate) were similar within all datasets (average range 3.4; Additional file 1: Table S1). There was no correlation in TIN-estimates between paired cancer and normal samples from the colon $(n=19)$, lung $(n=20)$, prostate $(n=29)$, or stomach $(n=23$; Additional file 1 : Figure S1). In the breast cancer dataset, there were significantly lower TIN-estimates in estrogen and progesterone receptor positive than negative samples $(P=0.04$, independent samples t-test; Additional file 1: Figure S2). Also, there were significant differences in TIN-estimates between histological subtypes of both cervical cancer and lung cancer series II. In the cervical cancer dataset, adenocarcinomas had higher TIN-estimates than squamous cell carcinomas $(P=0.002)$, whereas the opposite was found in lung cancer series II $(P<0.001$; Additional file 1: Figure $\mathrm{S} 2$ ). There were no associations between TIN-estimates and sample characteristics in any of the other datasets (characteristics listed in Table 1).

For samples in seven of the cancer datasets (breast cancer, cervical cancer, colorectal cancer series I and II, lung cancer series II, and prostate cancer series I and II), the sample-wise TIN-estimates correlated strongly with the expression levels of pre-mRNA splicing factors $(n=280)$. The amount of significantly correlated splicing factor genes (Pearson correlation, $P<0.05$ ) ranged from $41 \%$ (colorectal cancer series I) to $70 \%$ (prostate cancer series I) within the datasets (Figure 1a). There was a clear preference for negative correlation, and the average correlation coefficients for the significantly correlated genes ranged from -0.5 to -0.3 (colorectal cancer series I and II, respectively). In these datasets, samples were also separated according to TIN-estimates when performing unsupervised hierarchical clustering analyses by the expression levels of all splicing factors (Additional file 1: Figure S3). Principal components analyses based on splicing factor gene expression corroborated these results (Figure 1b). In general, samples with low TIN-estimates $(\leq-1.0)$ were separated from samples with high TIN-estimates $(\geq 1.0)$, although not perfectly in colorectal cancer series II and prostate cancer series I. This association between TINestimates and expression levels of splicing factors was less clear in the other three cancer datasets (gastric cancer, lung cancer series I, and neuroblastoma; Figure 1). Here, the amount of significantly correlated splicing factor genes 
Table 1 Samples included in the study

\begin{tabular}{|c|c|c|c|c|c|}
\hline Tissue & Samples & Histology & Other sample characteristics & $\begin{array}{l}\text { GEO accession } \\
\text { number }\end{array}$ & $\begin{array}{l}\text { Literature } \\
\text { references }\end{array}$ \\
\hline Breast cancer & 84 & - & $\begin{array}{l}\text { Hormone receptor status: HER2-positive } \\
(n=35) \text {; ER/PR-positive }(n=25) ; \text { ER/PR/ } \\
\text { HER2-negative }(n=24)\end{array}$ & GSE16534 & [45] \\
\hline Cervical cancer & 28 & $\begin{array}{l}\text { Squamous cell carcinoma }(n=19) \\
\text { adenocarcinoma }(n=9)\end{array}$ & - & GSE27388 & [44] \\
\hline $\begin{array}{l}\text { Colorectal cancer } \\
\text { series I }\end{array}$ & 26 & Adenocarcinoma & - & GSE16534 & [45] \\
\hline \multirow[t]{2}{*}{$\begin{array}{l}\text { Colorectal cancer } \\
\text { series II }\end{array}$} & $101^{\mathrm{a}}$ & Adenocarcinoma & $\begin{array}{l}\text { Stage: Stage I }(n=28) ; \text { stage } \|(n=34) \text {; } \\
\text { stage III }(n=26) ; \text { stage IV }(n=13)\end{array}$ & $\begin{array}{l}\text { GSE24550 } \\
(n=55) ; \text { GSE29638 } \\
(n=46)\end{array}$ & {$[20,49]$} \\
\hline & & & $\begin{array}{l}\text { MSI-status: MSI-high }(n=21) \text {; MSS/ } \\
\text { MSI-low }(n=77) ; N A(n=3)\end{array}$ & & \\
\hline Gastric cancer & 25 & Adenocarcinoma & $\begin{array}{l}\text { Stage: Stage I }(n=8) ; \text { stage II }(n=4) ; \\
\text { stage III }(n=5) ; \text { stage IV }(n=8)\end{array}$ & GSE13195 & - \\
\hline $\begin{array}{l}\text { Lung cancer } \\
\text { series I }\end{array}$ & 20 & Non-small-cell lung adenocarcinoma & $\begin{array}{l}\text { Stage: Stage I }(n=12) \text {; stage } \|(n=3) \text {; } \\
\text { stage III }(n=5)\end{array}$ & GSE12236 & [48] \\
\hline $\begin{array}{l}\text { Lung cancer } \\
\text { series II }\end{array}$ & 43 & $\begin{array}{l}\text { Non-small-cell lung adenocarcinoma }(n=21) \\
\text { and squamous cell carcinoma }(n=22)\end{array}$ & - & GSE16534 & [45] \\
\hline Neuroblastoma & 47 & - & Stage: stage I $(n=10)$; stage IV $(n=37)$ & GSE27608 & [43] \\
\hline $\begin{array}{l}\text { Prostate cancer } \\
\text { series I }\end{array}$ & 131 & $\begin{array}{l}\text { Adenocarcinoma Gleason grade: Grade } 5 \\
(n=1) \text {; grade } 6(n=77) ; \text { grade } 7(n=42), \\
\text { grade } 8(n=7) ; \text { grade } 9(n=4)\end{array}$ & $\begin{array}{l}\text { Pathologic T stage: stage } 2(n=85) \\
\text { stage } 3(n=40) ; \text { stage } 4(n=6)\end{array}$ & GSE21034 & [47] \\
\hline $\begin{array}{l}\text { Prostate cancer } \\
\text { series II }\end{array}$ & $50^{\mathrm{a}}$ & $\begin{array}{l}\text { Gleason grade: Grade } 5(n=2) \text {; grade } 6 \\
(n=15) \text {; grade } 7(n=32) \text {; grade } 9(n=1)\end{array}$ & $\begin{array}{l}\text { Pathologic T stage: stage } 2(n=26) \text {, } \\
\text { stage } 3(n=24)\end{array}$ & GSE42954 & {$[46]$} \\
\hline $\begin{array}{l}\text { Normal colonic } \\
\text { mucosa }\end{array}$ & $21^{\mathrm{a}}$ & & $\begin{array}{l}19 \text { samples corresponding to tumors } \\
\text { from GSE24550 and GSE29638 }\end{array}$ & $\begin{array}{l}\text { GSE42690 }(n=19) \\
\text { GSE29638 }(n=2)\end{array}$ & {$[20,49]$} \\
\hline Normal lung & 20 & & $\begin{array}{l}\text { Corresponding to tumors from } \\
\text { GSE12236 }\end{array}$ & GSE12236 & [48] \\
\hline Normal prostate & 29 & & $\begin{array}{l}\text { Corresponding to tumors from } \\
\text { GSE21034 }\end{array}$ & GSE21034 & [47] \\
\hline Normal stomach & $23^{b}$ & & $\begin{array}{l}\text { Corresponding to tumors from } \\
\text { GSE13195 }\end{array}$ & GSE13195 & - \\
\hline
\end{tabular}

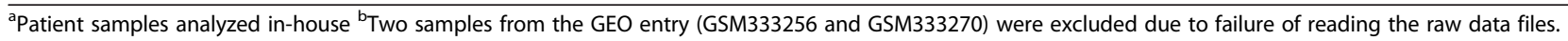
Abbreviations: GEO Gene Expression Omnibus, MSI microsatellite instability, MSS microsatellite stable, NA not available $T$ tumor.

ranged from $2 \%$ (lung cancer series I) to 10\% (neuroblastoma). Analyses of the datasets with normal samples indicated associations between TIN-estimates and expression levels of splicing factors also in non-cancerous tissues, with strongest associations in normal colonic mucosa (Additional file 1: Figure S4). This variance between the datasets showed no associations to quality control metrics of the exon microarray data (Additional file 1: Figures S5 and Additional file 1: Figure S6). The three cancer datasets not showing associations between TIN-estimates and expression levels of splicing factors had similar quality control metrics to the other datasets, whereas the dataset showing most deviance during quality control (cervical cancer), showed strong associations.

To test whether the observed associations between TIN-estimates and expression levels of splicing factors were greater than expected by chance, comparisons were made with random gene sets of equal size $(n=1,000$ sets of 280 genes; calculation of percentage of genes in each random gene set with expression levels significantly correlated with the TIN-estimates), and permutations of the TIN-estimates in each dataset $(n=1,000$ permutations; calculation of percentage of splicing factor genes with expression levels significantly correlated with each round of permutation). Corresponding with the results above, the same seven cancer datasets that showed strongest associations, also had higher amounts of splicing factor genes with expression levels significantly correlated with the TIN-estimates than was expected by chance $(P<0.001)$, as opposed to the other three cancer datasets (Figure 2). The same results were obtained also when analyzing correlation strengths (Additional file 1: Figure S7). Interestingly, all the normal tissue datasets also had higher percentages of splicing factor genes with expression levels that were significantly correlated with the TIN-estimates than expected by chance (Additional file 1: Figure S8). 


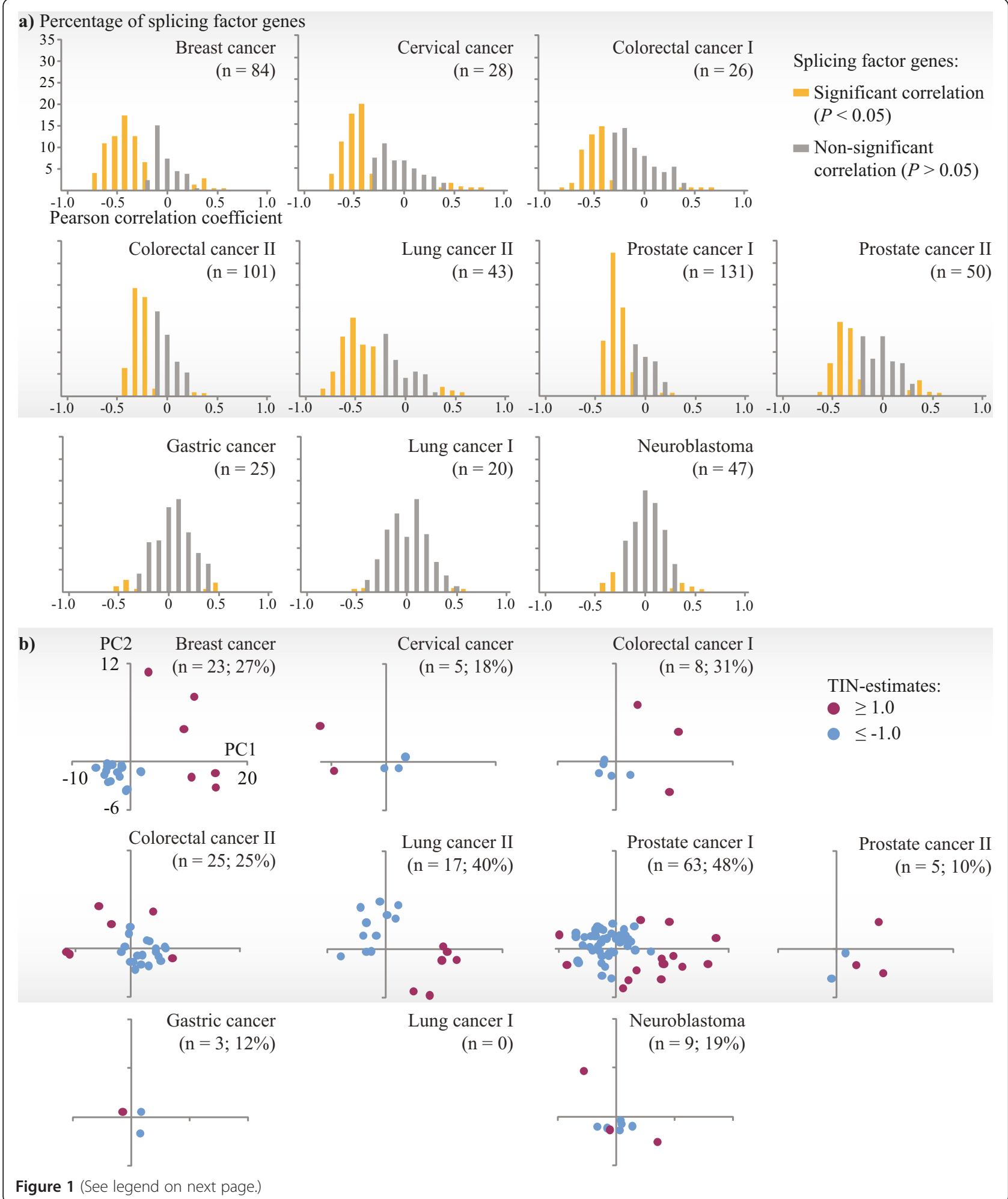


(See figure on previous page.)

Figure 1 TIN-estimates are associated with the expression levels of splicing factor genes in seven cancer datasets. (a) In seven of the cancer datasets (plots with grey background) there were strong and significant correlations (horizontal axes) between TIN-estimates and the expression levels of $\geq 41 \%$ of the totally 280 splicing factor genes. In the other three datasets, the correlations were mainly non-significant. Yellow and grey bars represent the percentages of splicing factor genes with significant and non-significant correlations, respectively. (b) In two-dimensional principal components analysis based on the expression levels of splicing factor genes $(n=280)$, samples were generally separated according to TIN-estimates (only samples with TIN-estimates $\geq \pm 1.0$ were included, the number and percentages of samples are indicated for each dataset) in the same seven datasets (with grey background). In colorectal cancer series II and prostate cancer series I, the separation was not complete. In the gastric cancer dataset and lung cancer series I, there were few samples with TIN-estimates $\geq \pm 1.0$. In the neuroblastoma dataset, there was no clear separation. PC, principal component.

The association was particularly strong in normal colonic mucosa, where $56 \%$ of the splicing factors were significantly correlated.

Also in comparison with all gene sets included in the Molecular Signatures Database v3.1 (MSigDB; annotated with Gene Ontology terms; $\mathrm{n}=1,454$ gene sets), as well as with the full genome ( $n=17,881$ genes), the splicing factor gene set (and other splicing-related gene sets included in MSigDB) had high amounts of genes with expression levels significantly correlated with TIN-estimates in the same seven cancer datasets (Additional file 1: Figure S9).

The percentage of TIN-samples (samples with TINestimates $\geq \pm 1.0$ ) in each dataset varied from 0 in lung cancer series I to $48 \%$ in prostate cancer series I (Figure 1b). The datasets with the highest percentages also had higher amounts of splicing factor genes with expression levels significantly correlated with the TIN-estimates (Additional file 1: Figure S10). We consider this to strengthen the notion that tissue and cancer types with high variation in splicing also have a correspondingly high variation in splicing factor expression.

\section{Inverse relationship between TIN-estimates and expression levels of splicing factors}

In the seven cancer datasets with strong associations between TIN-estimates and expression levels of splicing factors, the associations were mainly inverse. The amount of genes with expression levels that were negatively correlated with the TIN-estimates ( $\geq 89 \%$ of the significantly correlated splicing factor genes in all seven datasets) was much larger than the amount of positively correlated genes, with ratios ranging from 8 in prostate cancer series II to 97 in prostate cancer series I. This ratio of negative vs. positive correlations was significantly higher than expected by chance (again compared with random gene sets and permutations of the amounts of aberrant exon usage; $P<0.001$; Figure 3). This significant shift towards negative correlation was not found in the three cancer datasets with weak associations between TIN-estimates and expression levels of splicing factors (ratios ranging from 1 to 2 ; Figure 3). In the normal tissue types, there was also a significant, but less pronounced shift towards negative correlation. Here, the ratios of the amounts of significant negatively $v s$. positively correlated splicing factor genes ranged from 4 (normal colonic mucosa) to 30 (normal stomach; Additional file 1: Figure S11).

Also in comparison with gene sets in MSigDB, as well as with the full genome, the splicing factor gene set (and other splicing-related gene sets included in MSigDB) had a strong shift towards negative correlation in the same seven cancer datasets (Additional file 1: Figure S12).

\section{Transcriptome instability as a pan-cancer characteristic}

Although the individual normal tissue datasets showed strong correlations between the TIN-estimates and expression levels of splicing factors, this association was lost in pooled analyses of the normal tissues $(n=91)$ of four different origins. Again, this was tested by calculating the correlation between TIN-estimates and expression levels of splicing factors across all the different normal tissue types, and comparing with random gene sets and permutations of the TIN-estimates (Figure 4a). This is compliant with the previously described tissue specificity in both splicing patterns $[25,33]$ and expression levels of splicing factors reviewed in [34]. The same results were found from analysis of the paired cancernormal sample sets (except for in the colon; Additional file 1: Figure S13), again in agreement with the fact that pre-mRNA splicing is differentially regulated also between cancer and normal tissues [27]. Surprisingly, when doing the same comparisons across the cancer types with strong associations between TIN-estimates and splicing factor expression, the strong and non-random associations prevailed $(P<0.001$; Figure $4 \mathrm{~b}$; for this analysis, 20 samples were randomly selected from each of the five cancer types, to assess splicing patterns across similar numbers of samples as for the normal tissues. For cancer types represented by two datasets, samples were selected from the datasets with paired normal samples, i.e. colorectal cancer series II and prostate cancer series I). In these pan-cancer analyses, there was a much higher amount of significant negatively than positively correlated splicing factor genes (232 genes compared with 14 genes, ratio 17; also this higher than expected by chance, compared with random gene sets and permutations of the TIN-estimates, $P<0.001$; Figure $4 \mathrm{~b}$ ). When limiting 


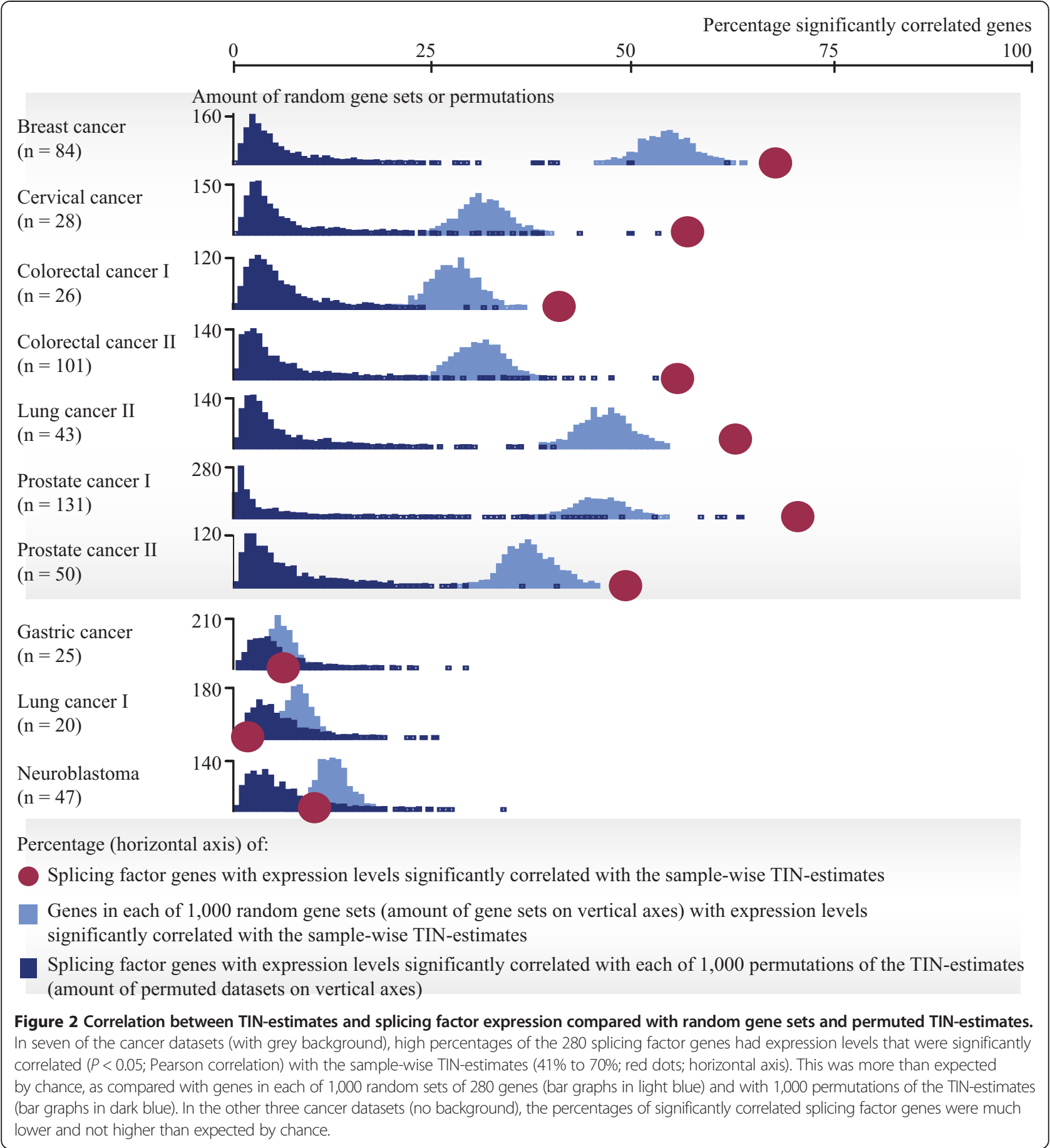

the analyses to cancer samples for which paired normal samples were available, the results were corresponding. Non-random associations between TIN-estimates and splicing factor expression were found only in analyses across colorectal cancer series II and prostate cancer series I (Additional file 1: Figure S13). This indicates that TIN is a shared molecular characteristic across the affected cancer types.

\section{Discussion}

We have previously proposed genome-wide instability of the transcriptome as a molecular, pre-mRNA splicing related characteristic of colorectal cancer. Here, we show data indicating that the transcriptomes of several, but not all, other types of solid tumors also have this feature. Common cancers of the breast, cervix, large bowel, lung, and prostate all show large variation in sample-wise 


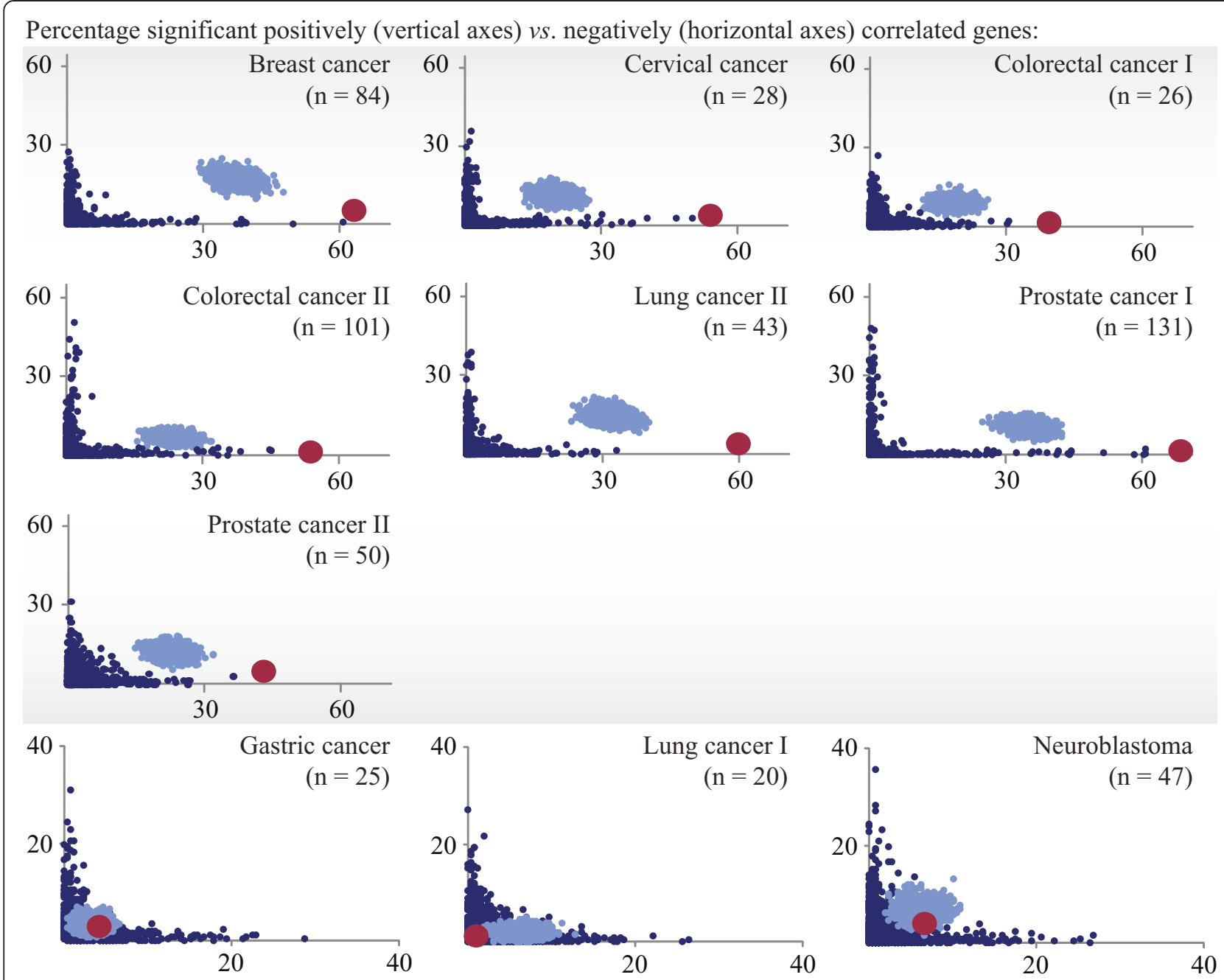

Percentage significantly correlated genes $(P<0.05$; Pearson correlation) comparing:

Expression levels of splicing factor genes and sample-wise TIN-estimates

Expression levels of genes in each of 1,000 random gene sets and sample-wise TIN-estimates

Expression levels of splicing factor genes and each of 1,000 permutations of the TIN-estimates

Figure 3 Negative correlation between TIN-estimates and splicing factor expression in the cancer datasets. In the seven cancer datasets (grey background) with strong associations between TIN-estimates and expression levels of splicing factors $(n=280)$, the relationship was inverse, with a much higher percentage of significant negatively (horizontal axes) than positively (vertical axes) correlated splicing factor genes (red). This shift was higher than expected by chance, as demonstrated by comparisons with genes in each of 1,000 random sets of 280 genes (light blue), and with each of 1,000 permutations of the TIN-estimates (dark blue). This significant shift towards negative correlation was not found in the three cancer datasets with weak associations between TIN-estimates and splicing factor expression (no background).

amounts of aberrant exon skipping and inclusion (TINestimates) that are significant negatively correlated with the expression levels of splicing factor genes. Such associations were also found within healthy tissue types, although to less extent. This is consistent with the fact that most tissues exhibit great and tightly regulated splicing variation. However, the splicing patterns were clearly distinct between healthy tissues and their malignant counterparts, as shown by discordant TIN- estimates between paired cancer and normal samples in all the four different tissue types analyzed. This is in compliance also with the original TIN-report, showing that colorectal cancers have higher TIN-estimates than normal colonic mucosa [20]. Furthermore, in analysis across the various healthy tissues, the correlation between TIN-estimates and expression levels of splicing factors was not greater than expected by chance. This indicates a failure to detect a common pattern of 


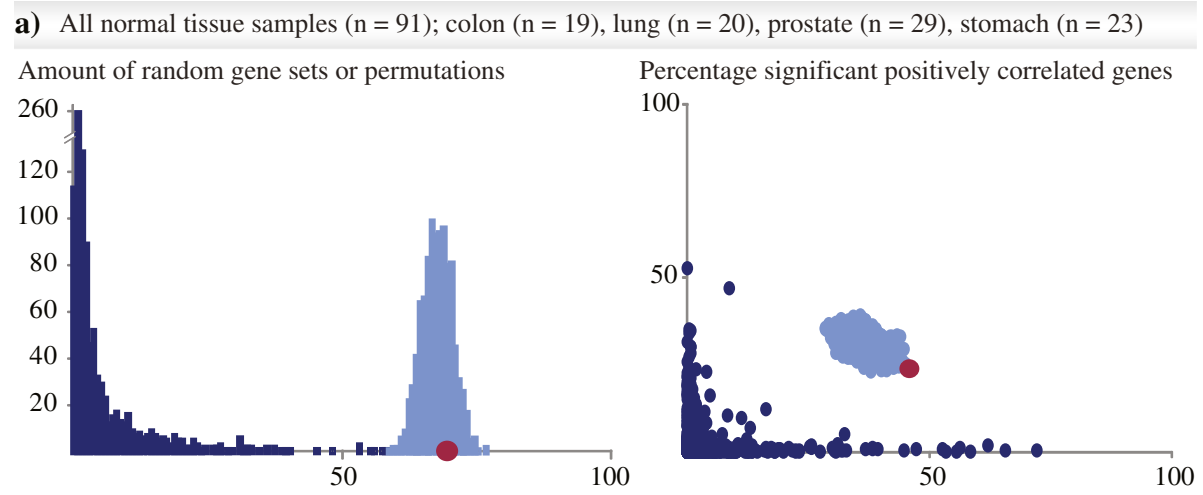

Percentage significantly correlated genes Percentage significant negatively correlated genes

b) All cancer types with transcriptome instability ( $\mathrm{n}=5 \times 20$ samples)
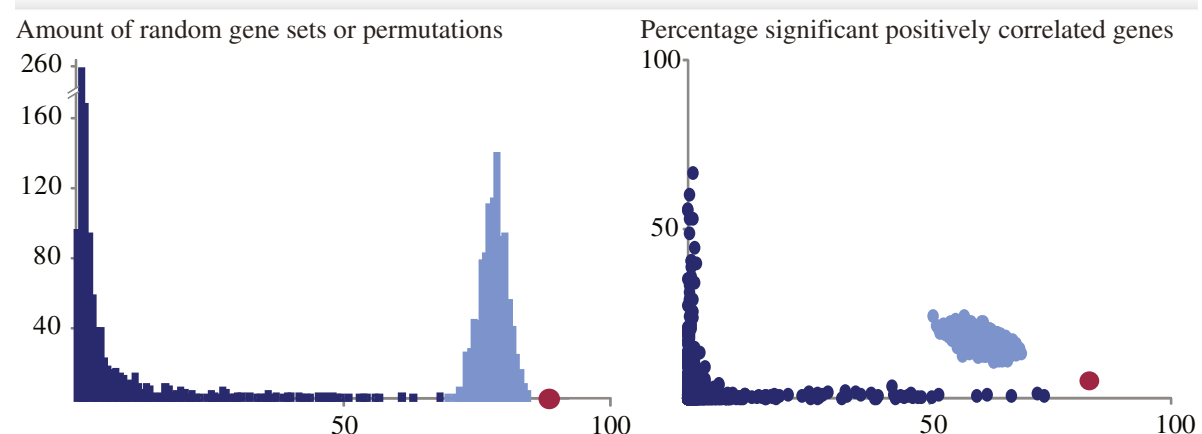

Percentage significantly correlated gene

Percentage significant negatively correlated genes

Percentage significantly correlated genes $(\mathrm{P}<0.05$; Pearson correlation $)$ comparing:

- Expression levels of splicing factor genes and sample-wise TIN-estimaes

Expression levels of genes in each of 1,000 random gene sets and sample-wise TIN-estimates

Expression levels of splicing factor genes and each of 1,000 permutations of the TIN-estimates

Figure 4 Correlation between TIN-estimates and splicing factor expression in pooled tissue types. The left and right plots correspond to plots in Figures 2 and 3, respectively. (a) In pooled analyses of samples from four different normal tissues, the percentage of splicing factor genes (totally 280 genes; red dots) with expression levels that were significantly correlated with the TIN-estimates $(P<0.05$; Pearson correlation), was not higher than expected by chance, as demonstrated by comparison with genes in each of 1,000 random sets of 280 genes (light blue) and with 1,000 permutations of the TIN-estimates (dark blue; left plot). The ratio between the amounts of significant negatively and positively correlated splicing factor genes was 2 (right plot). (b) Contrarily, across 20 randomly selected samples from each of five different cancer types with strong associations between TIN-estimates and splicing factor expression (breast cancer, cervical cancer, colorectal cancer series II, lung cancer series II, and prostate cancer I) the percentage of splicing factor genes with expression levels that were significantly correlated was higher than expected by chance (left plot). Also, there was a significant shift towards negative correlation (the ratio between the amounts of splicing factors genes with expression levels that were significant negatively and positively correlated was 17; right plot).

regulation, and that the splicing process is predominantly tissue specific in healthy tissues. Interestingly, in pan-cancer analyses, the strong associations with expression levels of splicing factors prevailed. These results suggest that TIN is a pan-cancer characteristic, clearly distinguished from normal splicing variation and splicing factor expression.

The importance of molecular pan-cancer analyses has recently been illuminated by The Cancer Genome Atlas
Pan-Cancer project [35]. The recognition that cancers from separate organs may have shared molecular features, while cancers from the same organ may be distinct, has great potential influence both biologically, on our understanding of the tumorigenic process, and clinically, for example for more personalized and targeted treatment strategies. Furthermore, pan-cancer analyses may aid in the identification of novel cancer-critical genes not disclosed in individual cancer types because of 
low mutation rates [36]. With regard to pan-cancer analysis of alternative splicing, mutation of the splicing factor gene $U 2 A F 1$ has been shown to result in both distinct and equal aberrant splicing events in lung adenocarcinoma and acute myeloid leukemia [37], indicating both specific and common regulation in the two cancer types. Here, from integrated expression analyses of a comprehensive collection of splicing factor genes, we have described TIN both within and across individual cancer types, suggesting that TIN is a general characteristic of cancer. Closer inspection of individual splicing factor genes revealed differences between the cancer types with respect to which genes were most closely associated with the aberrant splicing amounts (data not shown). No single splicing factor gene seceded in these pan-cancer analyses, suggesting that there is no dependency for the involvement of specific splicing factors in the establishment of TIN.

In this study, genome-wide analyses of alternative splicing were performed by computational analyses of exon-level microarray data. Although experimental work is needed to elucidate the functional mechanisms, an underlying biological explanation for the observed variation is suggested by the strong and non-random associations with the expression levels of splicing factors in several cancer types. In fact, this association increased with increasing splicing variation, as indicated by the correlation between the amount of TIN-samples per dataset (samples with TIN-estimates $\geq \pm 1.0$ ) and the number of splicing factor genes with expression levels that were significantly correlated with the TIN-estimates. Also worth noting is the striking inverse nature, with the great majority of splicing factors having expression levels that were negatively correlated with the TIN-estimates. Altogether, this suggests a mechanism in which decreased expression levels of splicing factors result in more aberrant exon skipping and inclusion. The fact that the majority of splicing factors are involved in several cancer types suggests a genome-wide mechanism. This is consistent with previous reports showing a coordinated regulation of RNA splicing by numerous splicing factors [38].

To describe TIN as a genuine molecular pan-cancer characteristic, identification of individual, aberrant splicing events and functional validation of their correlations with splicing factor expression levels are warranted. Such analyses are complicated by the genome-wide nature of the described associations. No single splicing factor is likely to account for all the observed variation. This situation resembles the obstacles faced also when characterizing the now well established genetic and epigenetic genome-wide phenotypes (with the exception of microsatellite instability found in subgroups of certain cancer types). However, we anticipate that ongoing RNA sequencing efforts will be highly valuable in gaining further insights into the proposed TIN characteristic.
There are various non-biological factors that may have influenced the splicing variation observed in this study. Firstly, the analyses are sensitive to the sizes of the datasets, and the variation in TIN-estimates increased with sample numbers (data not shown). This is an inherent consequence of the analyses, as differential exon skipping and inclusion were detected relative to all included samples. Secondly, technical variation in the microarray data may also have contributed. Accordingly, thorough quality control of the data was performed, and the extent of correlations between splicing variation and expression levels of splicing factors in each dataset, was independent of various quality control metrics. However, the failure to detect the strong association between splicing variation and splicing factor expression in three of the cancer datasets (gastric cancer, lung cancer series I, and neuroblastoma) may be attributable to non-biological features of the data rather than inherent characteristics of the cancer types. In particular, the two datasets from lung cancer showed conflicting results, with strong associations between the sample-wise TIN-estimates and splicing factor expression in series II, but not in series I. Both these patient series consist of non-small-cell carcinomas, but series I comprises adenocarcinomas only, whereas series II also includes squamous cell carcinomas. Although the TINestimates were significantly higher in the squamous cell carcinomas than the adenocarcinomas in lung cancer series II, the subtypes individually showed strong associations between the TIN-estimates and the expression levels of splicing factors (data not shown). For the other two cancer types represented by two datasets, colorectal cancer and prostate cancer, strong associations were clearly indicated in both datasets.

Splicing factor encoding genes have previously been nominated as cancer-critical based on their altered expression levels [28]. Recently, individual splicing factor genes have also been found to be commonly mutated in different cancer types, and this has been shown to have important implications for carcinogenesis [30-32,39,40]. In this study, mutation analyses have not been conducted. Furthermore, only the splicing events aberrant skipping and inclusion of cassette exons, as well as intron retention, have been considered. Although these are the most common modes of splicing [41], a complete view of the genome-wide effects of splicing factor expression on the splicing process should also consider the other events regulated by splicing factors (alternative $5^{\prime}$ and 3' splice sites, patterns of mutual exclusion, transsplicing, and exon scrambling).

Although premature, the implications of TIN as a common and novel, genome-wide characteristic of epithelial cancers are intriguing and warrant further investigation. Similarly to the genetic and epigenetic genome-wide phenotypes that have been proven to be important clinical 
characteristics, allowing for sub-grouping of patients according to prognosis $[13,16]$ and tumor histology [42], TIN may also have clinical potential. Previously, we have shown that TIN is associated with adverse outcome for patients with stage II and III colorectal cancer [20].

\section{Conclusions}

By computational analysis of alternative splicing based on exon-level microarray data, we show that common types of solid cancers (including breast, cervical, colorectal, lung, and prostate cancers) exhibit large variation among samples in amounts of aberrant skipping and inclusion that are non-randomly associated with the expression levels of splicing factors. Importantly, this type of transcriptome instability is a pan-cancer characteristic, as opposed to the tissue specificity prevailing in healthy tissues. Functional evaluation of the associations between this splicing pattern and the expression levels of splicing factors is needed to determine if transcriptome instability is a genuine phenotype of solid cancers.

\section{Methods \\ Patient material}

In this study, genome-wide expression data at the exon level for a comprehensive collection of 555 tissue samples from seven types of solid tumors, including the four most common, breast, colorectal, lung, and prostate, have been analyzed. Additionally, the study comprises expression data for a total of 93 normal tissue samples, including paired samples from cancers of the colon, lung, prostate, and stomach. All exon-level microarray datasets (Affymetrix HuEx-1_0-st-v2 arrays) with more than 20 cancer samples that have been made publically available by us and others have been included [20,43-49], except 83 colorectal cancer samples in GSE24549, which we previously used to describe TIN [20]. An overview of the datasets, with available clinical information of the tissue samples, is found in Table 1. From each of colorectal cancer, lung cancer and prostate cancer, two datasets have been included and are referred to as series I and II, respectively. Colorectal cancer series II is an in-house, consecutive series of stage I - IV cancers (90\% inclusion rate), collected at Aker University Hospital, Oslo, Norway, between 2005 and 2007. This series also comprises 21 normal colonic mucosa samples (including 6 that have not been published before) taken from disease-free areas distant to the primary tumors (19 matched sample pairs are included). Prostate cancer series II is also an in-house series, consisting of 50 primary tumor samples from a consecutive series of 200 clinically localized cancers treated with radical prostatectomy at the Portuguese Oncology Institute, Porto, Portugal.

The analysis of the additional samples published herein has been approved by the institutional review boards (the Regional Committee for Medical and Health Research Ethics, number 1.2005.1629; and reference [46]), which involves that informed consent is obtained from patients being enrolled to the study.

\section{Alternative splicing analysis}

All 648 samples have been analyzed for gene expression at the exon level by the Affymetrix GeneChip Human Exon 1.0 ST Array (Affymetrix Inc, Santa Clara, CA, USA). This array contains approximately 6 million different probes, providing genome-scale, exon-resolution expression measures. Each probe set consists of an average of four probes and generally corresponds to one exon. For each sample, 284,258 probe sets belonging to the 'core' set of probes targeting well annotated exons were analyzed, using an annotation file custom-made for alternative splicing analysis with the Finding Isoforms using Robust Multichip Analysis (FIRMA) algorithm (HuEx-1_0-st-v2,coreR3,A20071112,EP.cdf) [50,51].

Probe cell intensity (CEL) files storing raw data for each of the samples were used as input for preprocessing and alternative splicing analysis with the FIRMA algorithm implemented in the $\mathrm{R}$ software environment [52] (GEO accession numbers of both public and previously unpublished data are indicated in Table 1). This provided the basis for calculations of sample-wise amounts of aberrant exon skipping and inclusion within the datasets, as we have also previously described [20]. The first two steps of FIRMA follow the RMA approach [53] for background correction of individual probes based on GC-content, and for inter-chip quantile normalization. Then, alternative splicing scores (FIRMA scores) are calculated for each individual exon in each individual sample, as the deviance between the exon level and corresponding overall gene level expression measures. These scores represent the residuals from fitting gene level models to the exon level data, according to the mapping provided in the custommade annotation file. The FIRMA scores were log2transformed. Strong positive and negative scores reflect differential exon inclusion and skipping, respectively, compared with the rest of the samples in the dataset. The lower and upper $1^{\text {st }}$ percentiles of FIRMA scores across each dataset (Additional file 1: Table S1) were used as thresholds for identification of deviating (aberrant) exon skipping and inclusion, respectively. Sample-wise amounts of exons with aberrant splicing patterns were summarized from the exons exceeding these threshold values. Amounts of aberrant exon skipping or inclusion per sample are presented on a log2-scale, relative to the average sample-wise amount within the individual datasets. As a measure of TIN, aberrant exon skipping and inclusion were summarized. This TIN-estimate thus represents the total amounts of aberrant exon inclusion and skipping per sample (on a log2-scale), relative to the average amount 
within the dataset. TIN-estimates $\geq 1.0$, indicating twice as much aberrant exon skipping and inclusion as the average sample, and $\leq-1.0$, indicating half as much as the average sample, are used as a thresholds for characterizing samples with TIN (TIN-samples). In comparisons across datasets, alternative splicing analyses by the FIRMA algorithm, and calculation of sample-wise TIN-estimates, were done across all the samples included in each comparison, as indicated.

\section{Statistical analyses}

Statistical analyses were conducted in the $\mathrm{R}$ software environment (version 2.15.1). Permutations of TIN-estimates across the samples, and generation of random gene sets were done using the sample() function. Pearson correlations and two-sided Student $P$-values were calculated using the corAndPvalue() function in the WGCNA package [54]. Hierarchical clustering analyses with Euclidean distance metrics and complete linkage were done using J-Express 2011 (MolMine AS, Bergen, Norway). To corroborate the results from the clustering analyses, principle components analysis by singular value decomposition of the covariance matrix was performed using the $\operatorname{prcomp}()$ function in $\mathrm{R}$. The component scores for the first two principal components are used to illustrate twodimensional sample separation based on the input gene expression variables.

\section{Quality control of microarray data}

The analyses presented in this study are potentially sensitive to the quality of the microarray data. Hence, exon-level quality control of all included samples was performed (Supplementary Methods in Additional file 1). All cancer (Additional file 1: Figure S5) and normal (Additional file 1: Figure S6) samples were preprocessed together and compared using the quality assessment metrics recommended by Affymetrix [55] and reported by the Affymetrix Expression Console ${ }^{\mathrm{TM}}$ software.

\section{Splicing factors and miscellaneous gene sets}

We have created a comprehensive list of 280 splicing factor genes by combining search results from public annotation databases, as previously described [20]. Gene-level expression measures of these splicing factors were obtained from the CEL files of all samples included here. The CEL files were preprocessed across the datasets by the RMA approach [53], performing background correction, inter-chip quantile normalization, and gene-level summarization. For this, the Affymetrix Expression Console ${ }^{\mathrm{Tm}} 1.1$ software and Affymetrix HuEx-1_0-st-v2.r2 gene-core library files were used.

For comparison with the splicing factor genes, all the 1,454 Gene Ontology gene sets (the Gene Ontology Project [56]) collected in MSigDB [57] were also included.
This collection of gene sets (ranging in size from 10 to 2,131 genes) comprises 8,282 unique genes, corresponding to 7,923 transcript clusters on the exon arrays (matched by gene symbols), thus representing $44 \%$ of the genes on the arrays. These gene sets are available from the 'TIN' analysis package.

Previously unpublished microarray data have been deposited to the NCBI's Gene Expression Omnibus (GEO; accession numbers GSE42690 and GSE42954). R codes for alternative splicing analyses, statistical analyses, and plotting functions have been collected in a new Bioconductor package called 'TIN'. This package is available for download from http://www.bioconductor.org/. A description of the package, with analysis protocols and $\mathrm{R}$ codes can also be found at this web site.

\section{Availability of supporting data}

The data sets supporting the results of this article are available in the NCBI's Gene Expression Omnibus repository, GSE42690 and GSE42954.

\section{Additional file}

\section{Additional file 1: Supplementary Methods, Table S1, Figures S1-S13.}

\section{Abbreviations}

FIRMA: Finding isoforms using robust multichip analysis; GEO: Gene expression omnibus; mRNA: Messenger RNA; MSI: Microsatellite instability; MSigDB: The molecular signatures database; MSS: Microsatellite stable NA: Not available; T: Tumor; TIN: Transcriptome instability.

Competing interests

The authors declare that they have no competing interests.

\section{Authors' contributions}

AS and BJ carried out the data analyses. AS, MRT, RAL, and RIS participated in acquisition of the in-house and previously unpublished exon microarray data. AS drafted the manuscript. RIS conceived and directed the study. All authors participated in interpretation of results, contributed to manuscript preparation, and have read and approved the final version of the manuscript.

\section{Acknowledgements}

This work was funded by the Norwegian Health Region South-East [project numbers 2011024 to R.A.L. supporting A.S. as postdoc, 2012067 to R.I.S. supporting B.J. as PhD student]; the Norwegian Cancer Society [grant numbers PR-2006-0442 to R.A.L., PR-2007-0166 to R.I.S.]; and partly supported by the Research Council of Norway through its Centres of Excellence funding scheme [project number 179571]

\section{Author details}

${ }^{1}$ Department of Cancer Prevention, Institute for Cancer Research, The Norwegian Radium Hospital, Oslo University Hospital, P.O. Box 4953 Nydalen, Oslo NO-0424, Norway. ${ }^{2}$ Centre for Cancer Biomedicine, Faculty of Medicine, University of Oslo, P.O. Box 1078 Blindern, Oslo NO-0316, Norway.

${ }^{3}$ Department of Genetics, Portuguese Oncology Institute, Rua Dr. António Bernardino de Almeida, Porto 4200-072, Portugal. ${ }^{4}$ Cancer Genetics Group, Research Centre of the Portuguese Oncology Institute, Rua Dr. António Bernardino de Almeida, Porto 4200-072, Portugal. ${ }^{5}$ Institute of Biomedical Sciences Abel Salazar, University of Porto, Rua de Jorge Viterbo Ferreira n. ${ }^{\circ}$ 228, Porto 4050-313, Portugal.

Received: 25 April 2014 Accepted: 6 August 2014

Published: 10 August 2014 


\section{References}

1. Ferlay J, Shin HR, Bray F, Forman D, Mathers C, Parkin DM: Estimates of worldwide burden of cancer in 2008: GLOBOCAN 2008. Int J Cancer 2010, 127:2893-2917.

2. Lengauer C, Kinzler KW, Vogelstein B: Genetic instabilities in human cancers. Nature 1998, 396:643-649.

3. Hanahan D, Weinberg RA: Hallmarks of cancer: the next generation. Cell 2011, 144:646-674.

4. Fishel R, Lescoe MK, Rao MR, Copeland NG, Jenkins NA, Garber J, Kane M, Kolodner R: The human mutator gene homolog MSH2 and its association with hereditary nonpolyposis colon cancer. Cell 1993, 75:1027-1038.

5. Leach FS, Nicolaides NC, Papadopoulos N, Liu B, Jen J, Parsons R, Peltomaki P, Sistonen P, Aaltonen LA, Nystrom-Lahti M: Mutations of a mutS homolog in hereditary nonpolyposis colorectal cancer. Cell 1993, 75:1215-1225.

6. Fishel R, Kolodner RD: Identification of mismatch repair genes and their role in the development of cancer. Curr Opin Genet Dev 1995, 5:382-395.

7. Aaltonen LA, Peltomaki P, Leach FS, Sistonen P, Pylkkanen L, Mecklin JP, Jarvinen $\mathrm{H}$, Powell SM, Jen J, Hamilton SR: Clues to the pathogenesis of familial colorectal cancer. Science 1993, 260:812-816.

8. Ionov Y, Peinado MA, Malkhosyan S, Shibata D, Perucho M: Ubiquitous somatic mutations in simple repeated sequences reveal a new mechanism for colonic carcinogenesis. Nature 1993, 363:558-561.

9. Lothe RA, Peltomaki P, Meling Gl, Aaltonen LA, Nystrom-Lahti M, Pylkkanen L, Heimdal K, Andersen TI, Moller P, Rognum TO: Genomic instability in colorectal cancer: relationship to clinicopathological variables and family history. Cancer Res 1993, 53:5849-5852.

10. Lothe RA: Microsatellite instability in human solid tumors. Mol Med Today 1997, 3:61-68.

11. Peltomaki $P$, Lothe RA, Aaltonen LA, Pylkkanen L, Nystrom-Lahti M, Seruca R, David L, Holm R, Ryberg D, Haugen A: Microsatellite instability is associated with tumors that characterize the hereditary non-polyposis colorectal carcinoma syndrome. Cancer Res 1993, 53:5853-5855.

12. Merok MA, Ahlquist T, Røyrvik EC, Tufteland KF, Hektoen M, Sjo OH, Mala T, Svindland A, Lothe RA, Nesbakken A: Microsatellite instability has a positive prognostic impact on stage II colorectal cancer after complete resection: results from a large, consecutive Norwegian series. Ann Oncol 2012, 24:1274-1282.

13. Popat $S$, Hubner R, Houlston RS: Systematic review of microsatellite instability and colorectal cancer prognosis. J Clin Oncol 2005, 23:609-618.

14. Choi CM, Seo KW, Jang SJ, Oh YM, Shim TS, Kim WS, Lee DS, Lee SD: Chromosomal instability is a risk factor for poor prognosis of adenocarcinoma of the lung: Fluorescence in situ hybridization analysis of paraffin-embedded tissue from Korean patients. Lung Cancer 2009, 64:66-70.

15. Smid M, Hoes M, Sieuwerts AM, Sleijfer S, Zhang Y, Wang Y, Foekens JA, Martens JW: Patterns and incidence of chromosomal instability and their prognostic relevance in breast cancer subtypes. Breast Cancer Res Treat 2011, 128:23-30.

16. Walther A, Houlston R, Tomlinson I: Association between chromosomal instability and prognosis in colorectal cancer: A meta-analysis. Gut 2008 57:941-950

17. Pretorius ME, Waehre $H$, Abeler VM, Davidson B, Vlatkovic L, Lothe RA Giercksky KE, Danielsen HE: Large scale genomic instability as an additive prognostic marker in early prostate cancer. Cell Oncol 2009, 31:251-259.

18. Issa JP: CpG island methylator phenotype in cancer. Nat Rev Cancer 2004, 4:988-993.

19. Toyota M, Ahuja N, Ohe-Toyota M, Herman JG, Baylin SB, Issa JP: CpG island methylator phenotype in colorectal cancer. Proc Natl Acad Sci U S A 1999, 96:8681-8686.

20. Sveen A, Ågesen TH, Nesbakken A, Rognum TO, Lothe RA, Skotheim Rl: Transcriptome instability in colorectal cancer identified by exon microarray analyses: Associations with splicing factor expression levels and patient survival. Genome Med 2011, 3:32.

21. Barash Y, Calarco JA, Gao W, Pan Q, Wang X, Shai O, Blencowe BJ, Frey BJ: Deciphering the splicing code. Nature 2010, 465:53-59.

22. Salzman J, Gawad C, Wang PL, Lacayo N, Brown PO: Circular RNAs are the predominant transcript isoform from hundreds of human genes in diverse cell types. PLoS One 2012, 7:e30733.

23. Li H, Wang J, Mor G, Sklar J: A neoplastic gene fusion mimics trans-splicing of RNAs in normal human cells. Science 2008, 321:1357-1361.
24. Blencowe BJ: Alternative splicing: new insights from global analyses Cell 2006, 126:37-47.

25. Pan Q, Shai O, Lee LJ, Frey BJ, Blencowe BJ: Deep surveying of alternative splicing complexity in the human transcriptome by high-throughput sequencing. Nat Genet 2008, 40:1413-1415.

26. ENCODE Project Consortium, Bernstein BE, Birney E, Dunham I, Green ED, Gunter C, Snyder M: An integrated encyclopedia of DNA elements in the human genome. Nature 2012, 489:57-74.

27. Venables JP: Aberrant and alternative splicing in cancer. Cancer Res 2004 64:7647-7654.

28. Karni R, De Stanchina E, Lowe SW, Sinha R, Mu D, Krainer AR: The gene encoding the splicing factor SF2/ASF is a proto-oncogene. Nat Struct Mol Biol 2007, 14:185-193.

29. Skotheim Rl, Nees M: Alternative splicing in cancer: noise, functional, or systematic? Int J Biochem Cell Biol 2007, 39:1432-1449.

30. Yoshida K, Sanada M, Shiraishi Y, Nowak D, Nagata Y, Yamamoto R, Sato $Y$, Sato-Otsubo A, Kon A, Nagasaki M, Chalkidis G, Suzuki Y, Shiosaka M, Kawahata R, Yamaguchi T, Otsu M, Obara N, Sakata-Yanagimoto M, Ishiyama K, Mori H, Nolte F, Hofmann WK, Miyawaki S, Sugano S, Haferlach C, Koeffler HP, Shih LY, Haferlach T, Chiba S, Nakauchi H, et al: Frequent pathway mutations of splicing machinery in Myelodysplasia. Nature 2011, 478:64-69.

31. Imielinski $M$, Berger $A H$, Hammerman PS, Hernandez $B$, Pugh TJ, Hodis E, Cho J, Suh J, Capelletti M, Sivachenko A, Sougnez C, Auclair D, Lawrence MS, Stojanov P, Cibulskis K, Choi K, De WL, Sharifnia T, Brooks A, Greulich H, Banerji S, Zander T, Seidel D, Leenders F, Ansen S, Ludwig C, Engel-Riedel W, Stoelben E, Wolf J, Goparju C, et al: Mapping the hallmarks of lung adenocarcinoma with massively parallel sequencing. Cell 2012, 150:1107-1120

32. The Cancer Genome Atlas Network: Comprehensive molecular portraits of human breast tumours. Nature 2012, 490:61-70.

33. Wang ET, Sandberg R, Luo S, Khrebtukova I, Zhang L, Mayr C, Kingsmore SF, Schroth GP, Burge CB: Alternative isoform regulation in human tissue transcriptomes. Nature 2008, 456:470-476.

34. Chen M, Manley $\mathrm{J}$ : Mechanisms of alternative splicing regulation: insights from molecular and genomics approaches. Nat Rev Mol Cell Biol 2009, 10:741-754

35. Cancer Genome Atlas Research Network, Weinstein JN, Collisson EA, Mills GB, Shaw KR, Ozenberger BA, Ellrott K, Shmulevich I, Sander C, Stuart JM The cancer genome atlas pan-cancer analysis project. Nat Genet 2013, 45:1113-1120.

36. Gonzalez-Perez A, Perez-Llamas C, Deu-Pons J, Tamborero D, Schroeder MP, Jene-Sanz A, Santos A, Lopez-Bigas N: IntOGen-mutations identifies cancer drivers across tumor types. Nat Methods 2013, 10:1081-1082.

37. Brooks AN, Choi PS, De WL, Sharifnia T, Imielinski M, Saksena G, Pedamallu CS, Sivachenko A, Rosenberg M, Chmielecki J, Lawrence MS, DeLuca DS, Getz G, Meyerson M: A pan-cancer analysis of transcriptome changes associated with somatic mutations in U2AF1 reveals commonly altered splicing events. PLoS One 2014, 9:e87361.

38. Huelga SC, Vu AQ, Arnold JD, Liang TY, Liu PP, Yan BY, Donohue JP, Shiue L, Hoon S, Brenner S, Ares M Jr, Yeo GW: Integrative genome-wide analysis reveals cooperative regulation of alternative splicing by hnRNP proteins. Cell Rep 2012, 1:167-178

39. Quesada V, Conde L, Villamor N, Ordonez GR, Jares P, Bassaganyas L, Ramsay AJ, Bea S, Pinyol M, Martinez-Trillos A, Lopez-Guerra M, Colomer D, Navarro A, Baumann T, Aymerich M, Rozman M, Delgado J, Gine E, Hernandez JM, Gonzalez-Diaz M, Puente DA, Velasco G, Freije JM, Tubio JM, Royo R, Gelpi JL, Orozco M, Pisano DG, Zamora J, Vazquez $M$, et al: Exome sequencing identifies recurrent mutations of the splicing factor SF3B1 gene in chronic lymphocytic leukemia. Nat Genet 2011, 44:47-52

40. Wang L, Lawrence MS, Wan Y, Stojanov P, Sougnez C, Stevenson K, Werner L, Sivachenko A, DeLuca DS, Zhang L, Zhang W, Vartanov AR, Fernandes SM, Goldstein NR, Folco EG, Cibulskis K, Tesar B, Sievers QL, Shefler E, Gabriel S, Hacohen N, Reed R, Meyerson M, Golub TR, Lander ES, Neuberg D, Brown JR, Getz G, Wu CJ: SF3B1 and other novel cancer genes in chronic lymphocytic leukemia. $N$ Engl J Med 2011, 365:2497-2506.

41. Clark F, Thanaraj TA: Categorization and characterization of transcriptconfirmed constitutively and alternatively spliced introns and exons from human. Hum Mol Genet 2002, 11:451-464. 
42. Jass JR: Classification of colorectal cancer based on correlation of clinical, morphological and molecular features. Histopathology 2006, 50:113-130.

43. Guo X, Chen QR, Song YK, Wei JS, Khan J: Exon array analysis reveals neuroblastoma tumors have distinct alternative splicing patterns according to stage and MYCN amplification status. BMC Med Genomics 2011, 4:35.

44. Hall JS, Leong HS, Armenoult LS, Newton GE, Valentine HR, Irlam JJ, Moller-Levet C, Sikand KA, Pepper SD, Miller CJ, West CM: Exon-array profiling unlocks clinically and biologically relevant gene signatures from formalin-fixed paraffin-embedded tumour samples. Br J Cancer 2011, 104:971-981.

45. Lin E, Li L, Guan Y, Soriano R, Rivers CS, Mohan S, Pandita A, Tang J, Modrusan Z: Exon array profiling detects EML4-ALK fusion in breast, colorectal, and non-small cell lung cancers. Mol Cancer Res 2009, 7:1466-1476.

46. Paulo P, Ribeiro FR, Santos J, Mesquita D, Almeida M, Barros-Silva JD, Itkonen H, Henrique R, Jeronimo C, Sveen A, Mills IG, Skotheim RI, Lothe RA, Teixeira MR: Molecular subtyping of primary prostate cancer reveals specific and shared target genes of different ETS rearrangements. Neoplasia 2012, 14:600-611.

47. Taylor BS, Schultz N, Hieronymus H, Gopalan A, Xiao Y, Carver BS, Arora VK, Kaushik P, Cerami E, Reva B, Antipin Y, Mitsiades N, Landers T, Dolgalev I, Major JE, Wilson M, Socci ND, Lash AE, Heguy A, Eastham JA, Scher HI, Reuter VE, Scardino PT, Sander C, Sawyers CL, Gerald WL: Integrative genomic profiling of human prostate cancer. Cancer Cell 2010, 18:11-22.

48. Xi L, Feber A, Gupta V, Wu M, Bergemann AD, Landreneau RJ, Litle VR, Pennathur A, Luketich JD, Godfrey TE: Whole genome exon arrays identify differential expression of alternatively spliced, cancer-related genes in lung cancer. Nucleic Acids Res 2008, 36:6535-6547.

49. Ågesen TH, Sveen A, Merok MA, Lind GE, Nesbakken A, Skotheim RI, Lothe RA: ColoGuideEx: a robust gene classifier specific for stage II colorectal cancer prognosis. Gut 2012, 61:1560-1567.

50. Purdom E, Simpson KM, Robinson MD, Conboy JG, Lapuk AV, Speed TP: FIRMA: a method for detection of alternative splicing from exon array data. Bioinformatics 2008, 24:1707-1714.

51. Purdom E: HuEx-1_0-st-v2,coreR3,A20071112,EP.cdf. http://aroma-project.org/ data/annotationData/chipTypes/HuEx-1_0-st-v2/HuEx-1_0-st-v2,coreR3, A20071112,EP.cdf.gz.

52. R Core Team: $R:$ A language and environment for statistical computing. http://www.R-project.org/.

53. Irizarry RA, Hobbs B, Collin F, Beazer-Barclay YD, Antonellis KJ, Scherf U, Speed TP: Exploration, normalization, and summaries of high density oligonucleotide array probe level data. Biostatistics 2003, 4:249-264.

54. Langfelder P, Horvath S: WGCNA: an R package for weighted correlation network analysis. BMC Bioinformatics 2008, 9:559.

55. Affymetrix Inc: Quality assessment of exon and gene arrays. [http:/www.affymetrix. com/support/technical/whitepapers/exon_gene_arrays_qa_whitepaper.pdf]

56. Ashburner M, Ball CA, Blake JA, Botstein D, Butler H, Cherry JM, Davis AP, Dolinski K, Dwight SS, Eppig JT, Harris MA, Hill DP, Issel-Tarver L, Kasarskis A, Lewis S, Matese JC, Richardson JE, Ringwald M, Rubin GM, Sherlock G: Gene ontology: tool for the unification of biology. The Gene Ontology Consortium. Nat Genet 2000, 25:25-29.

57. Subramanian A, Tamayo P, Mootha VK, Mukherjee S, Ebert BL, Gillette MA, Paulovich A, Pomeroy SL, Golub TR, Lander ES, Mesirov JP: Gene set enrichment analysis: a knowledge-based approach for interpreting genome-wide expression profiles. Proc Natl Acad Sci U S A 2005, 102:15545-15550.

doi:10.1186/1471-2164-15-672

Cite this article as: Sveen et al:: Transcriptome instability as a molecular pan-cancer characteristic of carcinomas. BMC Genomics 2014 15:672.

\section{Submit your next manuscript to BioMed Central and take full advantage of:}

- Convenient online submission

- Thorough peer review

- No space constraints or color figure charges

- Immediate publication on acceptance

- Inclusion in PubMed, CAS, Scopus and Google Scholar

- Research which is freely available for redistribution

Submit your manuscript at www.biomedcentral.com/submit
Ciomed Central 